\title{
Curvaton and QCD Axion in Supersymmetric Theories
}

\author{
Eung Jin Chun ${ }^{1}$, Konstantinos Dimopoulos ${ }^{2,3}$ and David H. Lyth ${ }^{3}$ \\ ${ }^{1}$ Korea Institute for Advanced Study \\ 207-43 Cheongryangri-dong, Dongdaemun-Gu \\ Seoul 130-722, Korea \\ 2 Institute of Nuclear Physics, National Center for Scientific Research: 'Demokritos', \\ Agia Paraskevi Attikis, Athens 153 10, Greece \\ ${ }^{3}$ Physics Department, Lancaster University, Lancaster LA1 4YB, U.K.
}

April, 2004

\begin{abstract}
A pseudo Nambu-Goldstone boson as curvaton avoids the $\eta$-problem of inflation which plagues most curvaton candidates. We point out that a concrete realization of the curvaton mechanism with a pseudo Nambu-Goldstone boson can be found in the supersymmetric Peccei-Quinn mechanism resolving the strong $\mathrm{CP}$ problem. In the flaton models of Peccei-Quinn symmetry breaking, the angular degree of freedom associated with the QCD axion can naturally be a flat direction during inflation and provides successful curvature perturbations. In this scheme, the preferred values of the axion scale and the Hubble parameter during inflation turn out to be about $10^{10}$ $\mathrm{GeV}$ and $10^{12} \mathrm{GeV}$, respectively. Moreover, it is found that a significant isocurvature component, (anti)correlated to the overall curvature perturbation, can be generated, which is a smoking-gun for the curvaton scenario. Finally, non-Gaussianity in the perturbation spectrum at potentially observable level is also possible.
\end{abstract}




\section{Introduction}

The primordial curvature perturbation is caused presumably by some scalar field, which acquires its perturbation during inflation. For a long time it was generally agreed that the curvaturegenerating field would be the inflaton [1]. Then it was suggested instead that this field is some other 'curvaton' field [2, 3] (see also [4]), which generates the curvature perturbation only when its density becomes a significant fraction of the total. The curvaton proposal has received enormous attention because it opens up new possibilities both for model-building and for observation. ${ }^{1}$

In all cases, the field responsible for the curvature perturbation must be light during inflation, in the sense that its effective mass $m_{\text {eff }}$ is much less than the Hubble parameter $H$. (To be precise, we need during inflation $m_{\text {eff }}^{2} \lesssim 0.1 H^{2}$, so that the spectral tilt $1-n \simeq \frac{2}{3} m_{\text {eff }}^{2} / H^{2}$ satisfies the observational constraint.) If the responsible field is a curvaton, it should preferably also remain light after inflation, until $H$ falls below the true mass [7]. These requirements are in mild conflict with the generic expectation from supergravity, that the mass-squared of each scalar field will be at least of order $H^{2}$ [8]. This is the famous $\eta$-problem of inflation. Ways have been proposed to keep the responsible field sufficiently light [1], the most straightforward of them being to make it a pseudo Nambu-Goldstone boson (PNGB) (for the curvaton see [2, 9]).

For economy, and also to facilitate contact with observation, one would like a candidate for the responsible field to be one that is present in an already-existing model, designed for some purpose other than the generation of the curvature perturbation. Several such candidates have been proposed [9, 10, 11, 12, 13, but in general they do not come with a satisfactory mechanism for keeping the curvaton sufficiently light. The purpose of this paper is to suggest a natural curvaton candidate, which is present in flaton models of Peccei-Quinn symmetry breaking, and which is a PNGB.

The Peccei-Quinn (PQ) symmetry provides a nice solution to the strong CP problem [14. It is an anomalous global symmetry, $U(1)_{P Q}$, spontaneously broken at an intermediate scale $f_{P Q}$ with preferred value around $10^{12} \mathrm{GeV}$ providing enough dark matter (axion) of the universe. Its PNGB is the axion, which must be extremely light to satisfy the observational constraints [15]. In the context of supersymmetry (SUSY), two complex fields (or more) are needed to implement a global symmetry due to holomorphicity of the superpotential (unless the symmetry in question is R-symmetry). Hence, in SUSY, to spontaneously break the PQ symmetry one needs at least two complex fields, corresponding to two radial and two real angular degrees of freedom. One

\footnotetext{
${ }^{1}$ More recently still it has been suggested that the curvaton acts by causing inhomogeneous reheating [5] or through a preheating mechanism [6].
} 
of the latter is the axion, and the other is our curvaton candidate. ${ }^{2}$ We can define a symmetry (explicitly broken of course) acting only on the combination of phases which corresponds to the curvaton, and then the curvaton is the PNGB of that symmetry.

To understand how our model works, recall first that two fundamentally different paradigms exist for the PQ symmetry breaking fields. In one of them, the potentials involve only renormalizable terms in the superpotential plus small soft SUSY breaking terms, leading to a normal Mexican-hat potential whose height and width have the same scale $f_{P Q}$. According to this paradigm, PQ symmetry breaking persists in the limit of unbroken SUSY so that the axion has a well-defined supersymmetric scalar partner called the saxion (as well as a fermionic partner called the axino). The saxion is the only light degree of freedom apart from the axion, its mass coming from SUSY breaking and being of order TeV for gravity-mediated SUSY breaking. The other two degrees of freedom are heavy with mass of order $f_{P Q}$.

According to the other paradigm, the PQ symmetry breaking fields are instead flaton fields [16], so-called because they are symmetry-breaking fields, which correspond to flat directions of the potential (i.e., directions in which the quartic term is negligible). Their potential contains only soft SUSY breaking terms and non-renormalizable terms, which means that it is very flat. In the limit of unbroken SUSY there would be no spontaneous breaking of the PQ symmetry. A nice feature of this paradigm is that the intermediate axion scale $f_{P Q}$ is not put by hand but is generated through a geometric mean of the SUSY breaking scale and the Planck scale. The other degrees of freedom accompanying the axion get their mass only from SUSY breaking, making them of order $\mathrm{TeV}$ for gravity-mediated SUSY breaking. In this paper, we point out that this kind of model contains a natural curvaton candidate as the angular degree of freedom. The radial degrees of freedom are less suitable for this purpose, because in the early Universe they presumably acquire the mass of order $H$ which is generic [8] for scalar fields in a supergravity theory. In contrast, a mass of order $H$ is unlikely to be generated for the angular degree of freedom, because it would correspond to a generalized $A$-term which is forbidden if the fields responsible for the energy density are charged under certain symmetries [8]. We will analyze how the model parameters like the axion scale, the Hubble parameter and the curvaton decay temperature are constrained to produce successful curvature perturbations.

\footnotetext{
${ }^{2} \mathrm{By}$ 'degree of freedom' we mean as usual a normal mode of the coupled oscillations of the fields, which after quantization corresponds to a particle species.
} 


\section{Supersymmetric realization of the $\mathrm{PQ}$ and curvaton mechanisms}

The minimal model of the type that we are considering is a variation of the non-renormalizable superpotential [17, 18, 19], which introduces two fields $P$ and $Q$ charged under PQ symmetry and one singlet $S$ allowing the following superpotential

$$
W=h \frac{P^{n+1}}{M_{P}^{n}} H_{1} H_{2}+\lambda_{1} \frac{P Q S^{n+1}}{M_{P}^{n}}+\lambda_{2} \frac{S^{n+3}}{M_{P}^{n}}
$$

where the $U(1)_{P Q}$ and $Z_{N}$ charges are assigned as

$$
\begin{array}{rccccc} 
& H_{1} & H_{2} & P & Q & S \\
U(1)_{P Q}: & \frac{1}{2}(n+1) & \frac{1}{2}(n+1) & -1 & +1 & 0 \\
Z_{N}: & 1 & 1 & 1 & \alpha^{2} & \alpha
\end{array}
$$

with $\alpha \equiv e^{2 \pi i / N}$ and $N=n+3$. The field $S$ is supposed to have a negative soft mass-squared of order the gravitino mass $m_{3 / 2}$. This forces all the fields $P, Q$ and $S$ to develop the vacuum expectation values of the order

$$
v \sim\left(m_{3 / 2} M_{P}^{n}\right)^{1 / n+1}
$$

This sets the intermediate PQ scale

$$
f_{P Q} \sim 3 \times 10^{10}, \quad 1 \times 10^{13}, 3 \times 10^{14} \mathrm{GeV}
$$

for $n=1,2,3$, respectively, and $m_{3 / 2}=300 \mathrm{GeV}$. The negative mass-squared of the $S$ field can come from the renormalization effect [17] or from the initial condition of soft masses. We do not assume the negative mass-squared for the $P$ and $Q$ fields, which would drive the scalar potential unbounded from below when some of the fields $P, Q$ or $S$ are set to zero.

Note that the superpotential of Eq. (11) provides a natural solution to the $\mu$ problem 20] as the first non-renormalzable term leads to the right order of magnitude for the Higgs mass parameter;

$$
\mu=h \frac{v_{P}^{n+1}}{M_{P}^{n}} \sim m_{3 / 2} .
$$

The above interaction will be the main source of the decays of the particles corresponding to $P, Q$ or $S$ into the standard (s)particles.

The model under consideration contains six gauge singlet scalar particles. Three radial fields get masses of order $m_{3 / 2}$ from soft supersymmetry breaking. Among three phase fields, $\varphi_{P}$, 
$\varphi_{Q}$ and $\varphi_{S}$, one combination, $\varphi_{P}+\varphi_{Q}-2 \varphi_{S}$, gets the mass of order $m_{3 / 2}$ as the radial fields. The combination, $\varphi_{P}-\varphi_{Q}$, is the axion. The other combination is our curvaton candidate, $\varphi_{P}+\varphi_{Q}+\varphi_{S}$, which becomes massive due to the soft supersymmetry breaking $A$ term;

$$
V_{\text {soft }}=A \lambda_{1} \frac{P Q S^{n+1}}{M_{P}^{n}}+A \lambda_{2} \frac{S^{n+3}}{M_{P}^{n}}+\text { h.c. }
$$

inducing also in the vacuum a curvaton mass of order $m_{3 / 2}$. (For a detailed calculation in a similar model of the mass spectrum, see [21].) Note that, in our scheme, the curvaton parameters like its amplitude and decay temperature are fixed by the PQ scale and the $\mu$ term, as will be shown explicitly.

\section{The potential in the early Universe}

In order to consider the basic features of the curvaton field $\sigma$ associated with the QCD axion, let us simplify the original superpotential term in Eq. (II) as follows;

$$
W(\phi)=\frac{\lambda}{n+3} \frac{\phi^{n+3}}{M_{P}^{n}}
$$

where the complex field $\phi$ may be thought of as containing the phase field $\sigma$ and one radial component. This would be literally correct if the vacuum expectation values (VEVs) of $P, Q$ and $S$ happened to be equal, allowing the parameterization,

$$
\phi \equiv|\phi| e^{i \theta}=|\phi| \exp (i \sigma / \sqrt{2} v)
$$

with $v=\langle\phi\rangle$ and $\sigma \equiv \sqrt{2} v \theta$. (As noted in the Conclusion, our curvaton model can also be implemented using only $\phi$, though one then loses the connection with the QCD axion.)

With the above superpotential (5), the general scalar potential in the early universe can be written as

$$
V=\left(3 C_{\phi} H^{2}-m_{\phi}^{2}\right)|\phi|^{2}+\left[\left(C_{A} H+A\right) \frac{\lambda}{n+3} \frac{\phi^{n+3}}{M_{P}^{n}}+h . c .\right]+\lambda^{2} \frac{|\phi|^{2 n+4}}{M_{P}^{2 n}},
$$

where $m_{\phi}$ and $A$ are soft supersymmetry breaking masses at zero temperature. Note that we have put negative mass-squared for the $\phi$ field at zero temperature to generate the PQ scale as discussed previously.

The terms with $H$ are expected to arise from the supersymmetry breaking effect during and after inflation [8], except during radiation domination [22]. The generic expectation for $C_{\phi}$ is $\left|C_{\phi}\right| \sim 1$, and we are going to assume $C_{\phi} \sim-1$ so that there is PQ symmetry breaking. As 
is well known, a value $\left|C_{A}\right| \ll 1$ can be achieved provided that relevant field values are small on the Planck scale, if a symmetry forbids low-order terms in the Kähler potential which are linear in the field responsible for the energy density $[8]$. To be safe though, we need an estimate of the actual value of $C_{A}$, considering for completeness also $C_{\phi}$.

During inflation, the supergravity potential is taken to be generated by a single $F$-term $F_{I}$;

$$
V=e^{K / M_{\mathrm{P}}^{2}}\left(\left|F_{I}\right|^{2}-3 \frac{|W|^{2}}{M_{P}^{2}}\right)=3 H^{2} M_{P}^{2}
$$

where

$$
\left|F_{I}\right|^{2} \equiv\left(\partial_{I} W+W \partial_{I} K / M_{P}^{2}\right) K^{I I^{*}}\left(\partial_{I} W+W \partial_{I} K / M_{P}^{2}\right)^{*}
$$

and $K^{I I^{*}}$ is an element of the inverse of the Kähler metric $K_{I J *} \equiv \partial_{I} \partial_{J *} K$ with $J$ running over all of the scalar fields. Depending on the model, the field $I$ might be the inflaton, the waterfall field or some other field [1].

We make the following assumptions; (i) in the Kähler potential, terms linear in $I$ are negligible (this can be a consequence of a global or gauge symmetry), (ii) the field $I$ is much smaller than the Planck mass; $I \ll M_{P}$, as is true in most inflation models, and (iii) the field $\phi$ is also much smaller than $M_{P} ; \phi \ll M_{P}$. The last two conditions allow us to make a perturbative expansion of the potential in terms of $I$ and $\phi$. Under these assumptions, we write the most general Kähler potential and superpotential :

$$
\begin{aligned}
K & =I^{\dagger} I+\phi^{\dagger} \phi+\frac{c}{M_{P}^{2}} I^{\dagger} I \phi^{\dagger} \phi+\left[\frac{d}{M_{P}^{3}} I^{\dagger} I W(\phi)+h . c .\right] \\
W & =W(I)+W(\phi)
\end{aligned}
$$

neglecting terms with higher powers of $I^{\dagger} I, \phi^{\dagger} \phi$ and $W(\phi)$ which give more suppressed contributions. The conditions (ii) and (iii) imply that $F_{I} \approx \partial_{I} W \approx \sqrt{3} H M_{P}$ and $W / M_{P} \equiv \alpha I H \ll F_{I}$ with $\alpha \lesssim 1$. Then, we have

$$
C_{\phi} \approx(1-c), \quad C_{A} \approx \sqrt{3}[1-c(n+3)-\sqrt{3} \alpha] \frac{I}{M_{P}}-3 d \frac{H}{M_{P}} .
$$

As advertised, one obtains $\left|C_{\phi}\right| \sim 1$ but $\left|C_{A}\right| \ll 1[8]$.

If $H$ replaces $m_{\phi}$, Eq. (3) becomes $v \sim\left(H M_{P}^{n}\right)^{1 / n+1}$, which leads to the following;

$$
v \sim \begin{cases}\left(H M_{P}^{n}\right)^{1 / n+1} & \text { during inflation, } \\ {\left[\max \left(H, m_{3 / 2}\right) M_{P}^{n}\right]^{1 / n+1}} & \text { after inflation, } \\ \left(m_{3 / 2} M_{P}^{n}\right)^{1 / n+1}=f_{P Q} & \text { after reheating. }\end{cases}
$$


While the radial degree of freedom follows such a thermal history, the corresponding phase degree of freedom, $\sigma$, will enjoy the following potential of the pseudo Nambu-Goldstone boson (PNGB) type [9]:

$$
V(\sigma) \approx\left(C_{A} H+A\right) v^{3}\left(\frac{v}{M_{P}}\right)^{n}\left[1-\cos \left(\frac{\sigma}{v}\right)\right]
$$

which is very flat during and after inflation. From Eq. (12), one finds

$$
m_{\sigma}^{2} \sim\left(C_{A} H+A\right) \max \left\{H, m_{3 / 2}\right\} \sim \begin{cases}C_{A} H^{2} & \text { during inflation, } \\ \max \left(C_{A} H^{2}, A H, A m_{3 / 2}\right) & \text { after inflation, } \\ A m_{3 / 2} & \text { after reheating, }\end{cases}
$$

where we assumed that, during inflation $H_{*}>A / C_{A}$.

\section{The curvaton cosmology}

It is now obvious that the field $\sigma$ will naturally realize the curvaton paradigm. Taking Eq.(5) literally, $\sigma$ can be parameterized as $\theta \equiv \sigma / \sqrt{2} v$, that is, $\phi=v e^{i \theta}$ [cf. Eq. (6)]. To obtain simple predictions, let us assume that the value of $\sigma$ is completely randomized during inflation, so that in our part of the Universe $\theta$ at horizon exit has some value $\theta_{0}<2 \pi$. (We will come back to this point later).

It remains constant as far as $H \gg m_{\sigma}$ and the oscillation starts at $H \sim m_{3 / 2}$. Finally, the curvaton decays and thermalizes at $H \sim \Gamma_{\sigma}$ which is determined to be quite late due to its axion-like couplings. The interaction of $\sigma$ with ordinary particles is governed by the effective $\mu$ term in Eq. (10) leading to $\mu=h f_{P Q}^{n+1} / M_{P}^{n} \sim m_{3 / 2}$. Thus, the decay rate of $\sigma$ into two Higgs field is

$$
\Gamma_{\sigma} \approx \frac{(n+1)^{2}}{4 \pi} \frac{m_{3 / 2}^{3}}{f_{P Q}^{2}}
$$

where we put $\mu=m_{\sigma}=m_{3 / 2}$. Taking the approximation of $\Gamma_{\sigma} \sim m_{3 / 2}^{3} / f_{P Q}^{2}$ and $f_{P Q} \sim$ $\left(m_{3 / 2} M_{P}^{n}\right)^{1 / n+1}$, we get the decay temperature of $\sigma$ as follows;

$$
T_{\text {dec }} \sim\left\{\begin{array}{lc}
160 \mathrm{GeV} & \text { for } n=1 \\
0.6 \mathrm{GeV} & \text { for } n=2 \\
30 \mathrm{MeV} & \text { for } n=3
\end{array}\right.
$$

with $m_{3 / 2}=300 \mathrm{GeV}$. Here we have restricted ourselves to $n \leq 3$ for which the decay occurs well before the nucleosynthesis occurring around $T_{\mathrm{BBN}} \sim 1 \mathrm{MeV}$.

The density perturbation driven by the curvaton is given by [2]

$$
\zeta=\frac{1}{\pi} \frac{r}{4+3 r} \frac{H_{*}}{\sigma_{*}}
$$


where $r$ is the density ratio of $\rho_{\sigma} / \rho_{\gamma}$ at the time of the $\sigma$ decay:

$$
r \approx \frac{1}{6}\left(\frac{H_{\bar{m}}}{\Gamma_{\sigma}}\right)^{1 / 2}\left(\frac{\sigma_{*}}{M_{P}}\right)^{2}
$$

Here $H_{\bar{m}} \equiv \min \left(m_{\sigma}, \Gamma_{I}\right)$ [7] where $\Gamma_{I}$ is the inflaton decay rate determining the reheating temperature. Note that, in our model, the values of $r, H_{*}$ and $\sigma_{*}$ are determined by dimensionality of the superpotential in Eq. (5), which are related to the axion scale, $f_{P Q}$.

Inserting Eqs. (12) and (15) to Eqs. (17) and (18), we can determine the inflation Hubble parameter $H_{*}$ and the density ratio $r$ which reproduce the observed value of $\zeta \approx 5 \times 10^{-5}$. First, the value of $r$ can be calculated from the relation:

$$
\frac{r^{\frac{n+2}{2}}}{1+\frac{3}{4} r} \sim \frac{4 \pi \zeta \theta_{0}^{n+1}}{6^{n / 2}}\left(\frac{H_{\bar{m}}}{m_{3 / 2}}\right)^{\frac{n}{4}}\left(\frac{M_{P}}{m_{3 / 2}}\right)^{\frac{n^{2}}{2(n+1)}}
$$

and then it fixes the inflation Hubble parameter as

$$
\frac{H_{*}}{M_{P}} \sim\left(4 \pi \zeta \theta_{0}\right)^{\frac{n+1}{n}}\left(\frac{1+\frac{3}{4} r}{r}\right)^{\frac{n+1}{n}} .
$$

For a typical set of input parameters; $m_{3 / 2}=300 \mathrm{GeV}$ and $H_{\bar{m}}=1 \mathrm{GeV}$, we get the following values for $H_{*} / M_{P}$ and $r$;

$$
\left(\frac{H_{*}}{M_{P}}, r\right) \sim\left\{\begin{array}{lll}
\left(8 \times 10^{-7} \theta_{0}^{-2 / 3},\right. & \left.1.4 \theta_{0}^{4 / 3}\right) & \text { for } n=1 \\
\left(1 \times 10^{-5} \theta_{0}^{3 / 2},\right. & \left.6 \times 10^{5} \theta_{0}^{3}\right) & \text { for } n=2 \\
\left(4 \times 10^{-5} \theta_{0}^{4 / 3},\right. & \left.2 \times 10^{8} \theta_{0}^{8 / 3}\right) & \text { for } n=3
\end{array}\right.
$$

which is valid for the typical case of $\theta_{0} \lesssim 1$, i.e. $r \lesssim 1$ for $n=1$ and $r \gg 1$ for $n=2$, 3. The exact behaviors of $\left(H_{*} / M_{P}, r\right)$ depending on $\theta_{0}$ are shown in Figures 1, 2 and 3.

The value of $r$ has to be large enough to avoid excessive non-Gaussianity in the density perturbation spectrum. The constraint from the WMAP observations reads: $r>9 \times 10^{-3}[23$. Enforcing this constraint in Eq. (19) we obtain the bound

$$
\theta_{0}>\frac{0.232}{(103.3 \pi \zeta)^{1 /(n+1)}}\left(\frac{H_{\bar{m}}}{m_{3 / 2}}\right)^{-\frac{n}{4(n+1)}}\left(\frac{M_{P}}{m_{3 / 2}}\right)^{-\frac{1}{2}\left(\frac{n}{n+1}\right)^{2}}
$$

Suppose first that we live in a typical part of the Universe, then $\theta_{0} \sim \mathcal{O}(1)$ (see appendix for a discussion on the randomization of the curvaton field). In this case the value of $r$ is always large enough to avoid excessive non-Gaussianity. For $n=2,3, H_{*}$ becomes barely compatible with the limit $H_{*} / M_{P}<10^{-5}$ coming from the observational bound on the primordial tensor 
perturbation. (Actually, the curvaton model requires a somewhat smaller limit [24], assuming that inflation is of the slow-roll type.) The case with $n=1$ then gives most satisfactory result with $H_{*} \sim 10^{12} \mathrm{GeV}$. In this case, the curvaton decay temperature is $T_{\text {dec }} \sim 100 \mathrm{GeV}$ and the resulting entropy dumping is not significant (it is comparable to the preexisting one), so that the usual cosmological properties are retained. That is, the conventional dark matter candidate, the lightest neutralino or the axion with $f_{P Q}=3 \times 10^{10} \mathrm{GeV}$, is equally acceptable within our curvaton context, and the usual baryogenesis mechanism can also work. Note, also, that in this case we can have substantial non-Gaussianity in the density perturbation spectrum. Indeed, from Eq. (21) we readily obtain [27]

$$
f_{\mathrm{NL}}=\frac{5}{4 r} \simeq 0.9 \theta_{0}^{-4 / 3}
$$

The largest value of the above corresponds to the smallest possible value of $\theta_{0}$, which is determined by Eq. (20), when we demand that $H_{*} / M_{P}<10^{-5}$. With a little algebra it can be shown that $\left(\theta_{0}\right)_{\min } \simeq 0.34 \sqrt{r}$, which gives $\left(f_{\mathrm{NL}}\right)_{\max } \simeq \mathcal{O}(10)$. Such a value of $f_{\mathrm{NL}}$ may be observable in the near future.

In the above we assumed a reheating temperature $T_{\text {reh }} \sim 10^{9} \mathrm{GeV}$, which saturates the constraint of gravitino overproduction. This constraint is important, in the case when $n=1$, because the entropy production by the curvaton decay is not enough to dilute the gravitinos. However, a lower $T_{\text {reh }}$ corresponds to a smaller $H_{\bar{m}}$, which results in a smaller $r$, according to Eq. (19). This, in turn, results in a larger $H_{*}$ [cf. Eq. (20)], which again violates the upper bound on $H_{*}$. On the other hand, a higher $T_{\text {reh }}$ corresponds to the range $1 \mathrm{GeV}<H_{\bar{m}} \leq m_{3 / 2}$, which, for a given $n$, can increase $r$ somewhat. If $r \gg 1$ then the entropy production by the decay of the curvaton relaxes the gravitino constraint. However, as shown by Eq. (20), $H_{*}$ loses its sensitivity to a large $r$, even though, as can be seen in Figure $1, H_{*}$ may be reduced down to $10^{-7} M_{P}$ for $H_{\bar{m}} \sim m_{3 / 2} \cdot{ }^{3}$

Alternatively, we may allow the possibility that $\theta_{0}$ is untypically small in our region. Then the value of $H_{*}$ needed to generate the observed density perturbation is reduced by a factor $\theta_{0}^{\frac{n+1}{n}}$ for $n=2,3$. Now the cases $n=2,3$ become viable, but the $n=1$ is not because it violates the $H_{*} \lesssim 10^{-5} M_{\mathrm{P}}$ bound [cf. Eq. (21)]. Moroever, for $n=2$ and 3 we now have the fascinating possibility that the curvature perturbation has the observed magnitude only because we live

\footnotetext{
${ }^{3}$ Note, here, that there is also a lower bound on $H_{*}$, as was derived in 25]. In our case it can be shown that, because $v$ during inflation is different that in the vacuum, this bound reads $H_{*}>\left(v_{*} / f_{P Q}\right)^{4 / 5} 10^{-12} M_{P}$ [26]. Using, Eqs. (3) and (12) this bound can be recast as $H_{*}>\left[10^{-15(n+1)}\left(M_{P} / m_{3 / 2}\right)\right]^{\frac{4}{5 n+1}} M_{P}$. The tightest case corresponds to $n=1$, which gives $H_{*}>10^{-9} M_{P}$. Clearly, our results in Eq. (21) satisfy well this bound for $\theta_{0} \sim \mathcal{O}(1)$.
} 
in a special part of the Universe. (To be precise, the bound $H_{*} \lesssim 10^{-5} M_{\mathrm{P}}$ makes this state of affairs inevitable for $n=2,3$ ) This is actually a quite common outcome of curvaton models [9], and provides a good example of how a theoretical model can make anthropic considerations mandatory (in absence of a model to fix $\theta$ dynamically to a small value).

In this model, Peccei-Quinn symmetry is broken during inflation and is not restored. No axionic strings are formed, and axions are produced by the oscillation of the homogeneous axion field. Their density is 15 .

$$
\Omega_{\mathrm{a}}=(1 \text { to } 10)\left(\frac{f_{P Q}}{10^{12} \mathrm{GeV}}\right)^{1.2}\left(\frac{N \theta_{\mathrm{a}}}{\pi}\right)^{2} D,
$$

where $N$ is the multiplicity of the axion vacuum and $D$ is the dilution coming from entropy production after axion creation. (In our model, $D=1$ for the most interesting case $n=1$.) The uncertainties are big, but it is clear that the axions can be the dark matter $\left(\Omega_{\mathrm{a}}=0.25\right)$ for $\theta_{\mathrm{a}}$ roughly of order 1 .

The perturbation $\delta \theta_{\mathrm{a}}$ will generate a matter isocurvature perturbation $S_{\mathrm{m}}$, which should be $\lesssim 0.1 \zeta$ to be compatible with observation. It is given by

$$
\begin{aligned}
S_{\mathrm{m}} & =\frac{1}{3} \Omega_{\mathrm{a}} \frac{\delta \rho_{\mathrm{a}}}{\rho_{\mathrm{a}}} \\
& =\frac{2}{3} \Omega_{\mathrm{a}} \frac{\delta \theta_{\mathrm{a}}}{\theta_{\mathrm{a}}} .
\end{aligned}
$$

But $\delta \theta_{\mathrm{a}} \sim \delta \theta_{0}$ because both are generated from the vacuum fluctuation. Therefore, in view of Eq. (17), we find

$$
\frac{S_{\mathrm{m}}}{\zeta} \sim(1 \text { to } 10)\left(\frac{f_{P Q}}{10^{12} \mathrm{GeV}}\right)^{1.2}\left(\frac{N}{\pi}\right)^{2} \theta_{\mathrm{a}} \theta_{0}\left(\frac{4+3 r}{3 r}\right) D
$$

which can be $\lesssim 0.1$ as required by present observation. On the other hand it could be observable in the future.

\section{Discussion and Conclusions}

Within the curvaton paradigm, we have proposed a new candidate for the field which causes the curvature perturbation. We believe that it is one of the most attractive candidates yet proposed for that field. Being a field that is part of the flaton realization of PQ symmetry-breaking, and it can easily be kept light during and after inflation. In a typical part of the universe, the observed magnitude $\sim 10^{-5}$ of the curvature perturbation comes from a modest and quite reasonable 
hierarchy between the inflationary Hubble parameter and the vacuum expectation value of the Peccei-Quinn field. The predicted values of the axion scale and the Hubble parameters are $10^{10}$ and $10^{12} \mathrm{GeV}$, respectively, corresponding to $n=1$. In this case, the entropy dumping due to the curvaton decay is negligible so that the conventional cosmological predictions concerning dark matter components and baryogenesis remain unchanged. The model can generate two kinds of isocurvature pertubations. One is an axion isocurvature perturbation, uncorrelated with the curvature perturbation. The other is an isocurvature perturbation in some other kind of dark matter, or in the baryonic matter, which is produced either before or at curvaton decay. As described in [27, 28, such a perturbation is generic to curvaton models, and is fully correlated or anti-correlated with the curvature perturbation. ${ }^{4}$ The detection of any such isocurvature perturbation would be evidence in support of the curvaton model.

Finally, we have shown that, in this case, non-Gaussianity at at an observable level (with $\left.f_{\mathrm{NL}} \sim \mathcal{O}(10)\right)$ is possible. Our curvaton candidate is a PNGB, corresponding to an angular degree of freedom associated with the QCD axion. As a consequence, it can avoid the usual mass of order $H$ during (and after) inflation, because supersymmetry breaking affects the mass only through $A$ terms, which can be controlled by appropriate symmetries.

Our model of PQ symmetry breaking is similar to one already extensively investigated 21, 29], except that we take the PQ symmetry to be spontaneously broken throughout the history of the Universe. In contrast, the investigations of [21, 29] assume that the symmetry is initially unbroken, leading to thermal inflation. These possibilities correspond respectively to radial masses-squared of order $\mp H^{2}$, and the two signs should be deemed equally likely in the absence so far of a string-theoretic prediction. The unbroken paradigm has a very different cosmology from the one that we are adopting, producing in particular copious saxion- or axinolike particles which may decay into relativistic axions whose energy density is enough to affect nucleosynthesis 21. Also, the lightest axino-like particle may be the lightest supersymmetric particle [29], providing a more natural implementation of the Cold Dark Matter scenario that was originally proposed [30] in the context of non-flaton models. None of this occurs within our paradigm.

As it invokes the axion, our model, as it stands, is open to the criticism that the axion mass is implausibly small. Indeed, in the kind of models that we have considered where the axion is an angular part of a complex field, a non-renormalizable term in the potential with dimension $d$ generically breaks PQ symmetry and contributes to the axion mass an amount

\footnotetext{
${ }^{4}$ Correlation or anti-correlation of the CDM \{baryon\} isocurvature perturbation depends on whether CDM creation \{baryogenesis\} takes place (just) before curvaton decay or due to the curvaton decay itself. Note, that there is no residual isocurvature perturbation if CDM creation \{baryogenesis\} occurs after curvaton decay.
} 
of order $\sim v^{(d-2) / 2}$ in Planck units. To keep the axion mass to the required value of order $10^{-30} M_{\mathrm{P}}$, terms up to $d \sim 12$ must respect the PQ symmetry. (We take $v \sim f_{P Q}=10^{12} \mathrm{GeV}$ for an estimate.) A widely-discussed possibility to ensure this is to impose a $Z_{n}$ subgroup of the PQ symmetry $32 .{ }^{5}$

Because of these considerations, one may favour a solution of the $\mathrm{CP}$ problem in which the QCD axion is identified with a string axion [31, or one without any axion at all. ${ }^{6}$ In that case the field considered in our proposal becomes ad hoc, introduced solely to explain the curvature perturbation. Still, because of its simplicity and the ease with which it is made sufficiently light, we feel that the present curvaton model is extremely attractive in comparison with others. Let us end by mentioning those other models which have a close connection with ours.

The closely related models are considered in Refs. [12, 13, where as in our case the effect of the angular part of a flat direction has been considered. The difference from our case is that the flat direction is supposed to have zero VEV, so that the curvaton does not exist as a particle in the vacuum. As a consequence, the phase can only induce the curvature fluctuations in the radial field which leads to different predictions. In addition, the proposal of [12] does not invoke the mass-squared of order $-H^{2}$, so that the radial potential is very flat with only a two-loop thermal correction breaking the symmetry. (In both cases, successful curvature perturbations can arise for $n=3$, contrary to our case with $n=1$.) Because of such features the success of these models depends on computations which are much more tricky than in our case, though that is of course not necessarily an argument against them. However, we note that the computation of [12, involving the very flat radial potential, works only if inflation lasts for a limited amount of time, because it takes the radial field during inflation to be at the edge of the slow-roll regime, $V^{\prime \prime} \sim H^{2}$. It seems more reasonable to assume that inflation lasts long enough to allow the quantum fluctuation to randomize the radial field within the smaller region $V \lesssim H^{4}$. (The analogous assumption for the angular field is of course the one that we made.)

\footnotetext{
${ }^{5}$ Imposing the full PQ symmetry of course works, but exact continuous global symmetries are widely regarded as incompatible with string theory and even the existence of gravity. A different possibility, which does not seem to have been mentioned before, might be to suppose that the PQ fields are actually moduli, making the origin a point of enhanced symmetry. Then the non-renormalizable terms may be suppressed by a factor $\left(\mathrm{TeV} / M_{\mathrm{P}}\right)^{2} \sim 10^{-30}$. However this is small enough for all $d \geq 5$ only if $f_{P Q}$ has an implausibly small value of order $10^{8} \mathrm{GeV}$. In any case, this mechanism cannot be used in the flaton case, which invokes an unsuppressed coefficient for one term.

${ }^{6}$ The string axion as a curvaton candidate is discussed in [9]. As the string axion is a PNGB one may be hopeful that it can be kept sufficiently light in the early Universe, but string phenomenology is not yet sufficiently developed that one can be sure.
} 
The other related model [1] also invokes a flaton model of PQ symmetry breaking, but now the curvaton candidate is a radial field and it is not clear how to keep it light in the early Universe. Also, the model works only if inflation is of short enough duration that the curvaton does not enter the randomization regime.

Acknowledgments: EJC was supported by the Korea Research Foundation Grant, KRF2002-070-C00022.

\section{A Appendix: Randomization of the PNGB curvaton}

In this appendix we elaborate more on the assumption of the complete randomization of the value of the curvaton during inflation. Since the curvaton is an effectively massless field during inflation, its value is perturbed by the action of quantum fluctuations, which introduce a perturbation of the order of $\delta \sigma=T_{H}$ per Hubble time, where $T_{H}=H_{*} / 2 \pi$ is the Hawking temperature of de-Sitter space. Provided that the potential $V(\sigma)$ is not too steep, these quantum 'kicks' can move the field around by random walk so that, given enough e-foldings, the phase $\theta_{0}$ may assume an arbitrary value (typically of order unity) by the time when the cosmological scales exit the horizon. The criterion for this to occur for a PNGB curvaton is $H_{*} \geq H_{c}$, where

$$
H_{c} \equiv \sqrt{m_{\sigma} v}
$$

The above critical value has been obtained by considering the fact that the region of $V(\sigma)$, where the field is randomized, is determined by the condition $V(\sigma) \lesssim H_{*}^{4}[33$. This condition can be understood as follows: The kinetic energy density of the quantum 'kicks' is $T_{H}^{4} \sim H_{*}^{4}$. Hence, the quantum fluctuations can displace the field from its minimum only up to potential density $V \sim T_{H}^{4}$. For a PNGB $V(\sigma) \sim m_{\sigma}^{2} \sigma^{2}$, which means that the borders of the randomized region are at $\sigma_{Q} \sim H_{*}^{2} / m_{\sigma}$. If $H_{*}$ is large enough then the randomized region includes the entire range of $\sigma$. Setting $\sigma_{Q} \rightarrow v$ we obtain the critical value of $H_{*}$, shown in Eq. (28), over which the PNGB is fully randomized.

Using Eqs. (12) and (14) the bound $H_{*} \geq H_{c}$ translates into

$$
C_{A} \leq\left(\frac{H_{*}}{M_{P}}\right)^{\frac{2 n}{n+1}} \sim\left(3 \pi \theta_{0} \zeta\right)^{2},
$$

where we also used Eq. (20) with $r \gtrsim 1$, according to Eq. (21). With $\theta_{0} \sim \mathcal{O}(1)$ we see that complete randomization of $\sigma_{*}$ requires $C_{A}<10^{-7}$. According to Eq. (11), this condition is satisfied provided that $|I| \lesssim 10^{-7} M_{\mathrm{P}}{ }^{7}$

\footnotetext{
${ }^{7}$ Note that, in this case, there is no problem with $C_{A} \geq A / H_{*} \sim 10^{-10}$.
} 
If complete randomization is not realized then $\sigma_{*}$ may be much smaller than $v$ during inflation. In this case, according to Eq. (18), $r$ can be smaller than unity and the results in Eq. (21) are modified. In particular, the typical value of $\sigma_{*}$ is estimated as

$$
\sigma_{*} \simeq \theta_{0} v \sim \begin{cases}v & \text { for } \quad H_{*} \geq H_{c} \\ H_{*}^{2} / m_{\sigma} & \text { for } \quad H_{*}<H_{c}\end{cases}
$$

where we considered that, in the non-randomized case, the typical value is $\sigma_{*} \sim \sigma_{Q}$. Inserting the above into Eq. (17) and after some algebra it is easy to find

$$
\tilde{r} \equiv \frac{r}{4+3 r}=\pi \zeta \sqrt{\frac{v}{m_{\sigma}}} \times\left\{\begin{array}{lll}
H_{c} / H_{*} & \text { for } \quad H_{*} \geq H_{c} \\
H_{*} / H_{c} & \text { for } \quad H_{*}<H_{c}
\end{array}\right.
$$

It it easy to see that $\tilde{r}(r)$ is an increasing function of $r$. Hence, the WMAP non-Gaussianity constraint reads: $\tilde{r}(r)>\tilde{r}\left(9 \times 10^{-3}\right) \sim 2 \times 10^{-3}$. For a successful PNGB curvaton, this constraint has to be satisfied at least for the maximum allowed $\tilde{r}$. Therefore, in view of Eq. (31), we obtain

$$
\tilde{r}_{\max } \simeq \pi \zeta \sqrt{v / m_{\sigma}}>2 \times 10^{-3}
$$

Using Eqs. (12), (14) and (20), we get

$$
\tilde{r}_{\max }^{2} \simeq \frac{\pi \zeta}{\sqrt{C_{A}} \theta_{0}} \tilde{r} \Rightarrow \tilde{r}_{\max }<\frac{\pi \zeta}{\sqrt{C_{A}} \theta_{0}}
$$

Combining Eqs. (32) and (33) we obtain

$$
\sqrt{C_{A}}<\frac{\pi \zeta}{\left(2 \times 10^{-3}\right) \theta_{0}} \simeq 0.08 \theta_{0}^{-1}
$$

which is typically satisfied by a successful curvaton.

\section{References}

[1] For a review, see D. H. Lyth and A. Riotto, Phys. Rept. 314, 1 (1999).

[2] D.H. Lyth and D. Wands, Phys. Lett. B524, 5 (2002).

[3] T. Moroi and T. Takahashi, Phys. Lett. B 522, 215 (2001) [Erratum-ibid. B 539, 303 (2002)].

[4] S. Mollerach, Phys. Rev. D 42, 313 (1990); A. D. Linde and V. Mukhanov, Phys. Rev. D 56, 535 (1997). 
[5] G. Dvali, A. Gruzinov and M. Zaldarriaga, astro-ph/0303591 L. Kofman, astro-ph/0303614.

[6] M. Bastero-Gil, V. Di Clemente and S. F. King, arXiv:hep-ph/0311237.

[7] K. Dimopoulos, G. Lazarides, D.H. Lyth and R. Ruiz de Austri, Phys. Rev. D 68, 123515 (2003).

[8] M. Dine, L. Randall, S. Thomas, Nucl. Phys. B458, 291 (1996); Phys. Rev. Lett. 75 (1995) 398.

[9] K. Dimopoulos, D.H. Lyth, A. Notari and A. Riotto, JHEP 0307, 053 (2003).

[10] A. Hebecker, J. March-Russell and T. Yanagida, Phys. Lett. B 552 (2003) 229; J. McDonald, Phys. Rev. D 68 (2003) 043505; M. Postma and A. Mazumdar, hep-ph/0304246; M. Bastero-Gil, V. Di Clemente and S. F. King, Phys. Rev. D 67 (2003) 103516; Phys. Rev. D 67, 083504 (2003).

[11] K. Dimopoulos, G. Lazarides, D.H. Lyth and R.R. de Austri, JHEP 0305, 057 (2003).

[12] K. Kasuya, M. Kawasaki and F. Takahashi, Phys. Lett. B578, 259 (2004).

[13] J. McDonald, hep-ph/0310126.

[14] R.D. Peccei and H.R. Quinn, Phys. Rev. Lett. 38, 1440 (1977); Phys. Rev. D16, 1791 (1977).

[15] For a review, see J.E. Kim, Phys. Rep. 150, 1 (1987).

[16] K. Yamamoto, Phys. Lett. B 161, 289 (1985).

[17] H. Murayama, H. Suzuki and T. Yanagida, Phys. Lett. B291, 418 (1992).

[18] K. Choi, E.J. Chun and J.E. Kim, Phys. Lett. B403, 209 (1997).

[19] G. Lazarides and Q. Shafi, Phys. Lett. B489, 194 (2000).

[20] J.E.Kim and H.P. Nilles, Phys. Lett. B138, 150 (1984); E.J. Chun, J.E. Kim and H.P. Nilles, Nucl. Phys. B370, 105 (1992).

[21] E.J. Chun, D. Comelli and D.H. Lyth, Phys. Rev. D62, 095013 (2000).

[22] D. H. Lyth and T. Moroi, JHEP 0405, 004 (2004).

[23] E. Komatsu et al., astro-ph/0302223.

[24] K. Dimopoulos and D. H. Lyth, arXiv:hep-ph/0209180.

[25] D. H. Lyth, Phys. Lett. B 579 (2004) 239.

[26] K. Dimopoulos, D. H. Lyth and Y. Rodriquez-Garcia, in preparation. 
[27] D. H. Lyth, C. Ungarelli and D. Wands, Phys. Rev. D 67, 023503 (2003).

[28] D. H. Lyth and D. Wands, Phys. Rev. D 68, 103516 (2003).

[29] E. J. Chun, H. B. Kim and D. H. Lyth, Phys. Rev. D 62, 125001 (2000).

[30] L. Covi, J. E. Kim and L. Roszkowski, Phys. Rev. Lett. 82, 4180 (1999).

[31] T. Banks and M. Dine, Nucl. Phys. B 505, 445 (1997).

[32] E.J. Chun and A. Lukas, Phys. Lett. B297, 298 (1992); G. Lazarides, C. Panagiotakopoulos and Q. Shafi, Phys. Rev. Lett. 56, 432 (1986).

[33] A. A. Starobinsky and J. Yokoyama, Phys. Rev. D 50, 6357 (1994). 

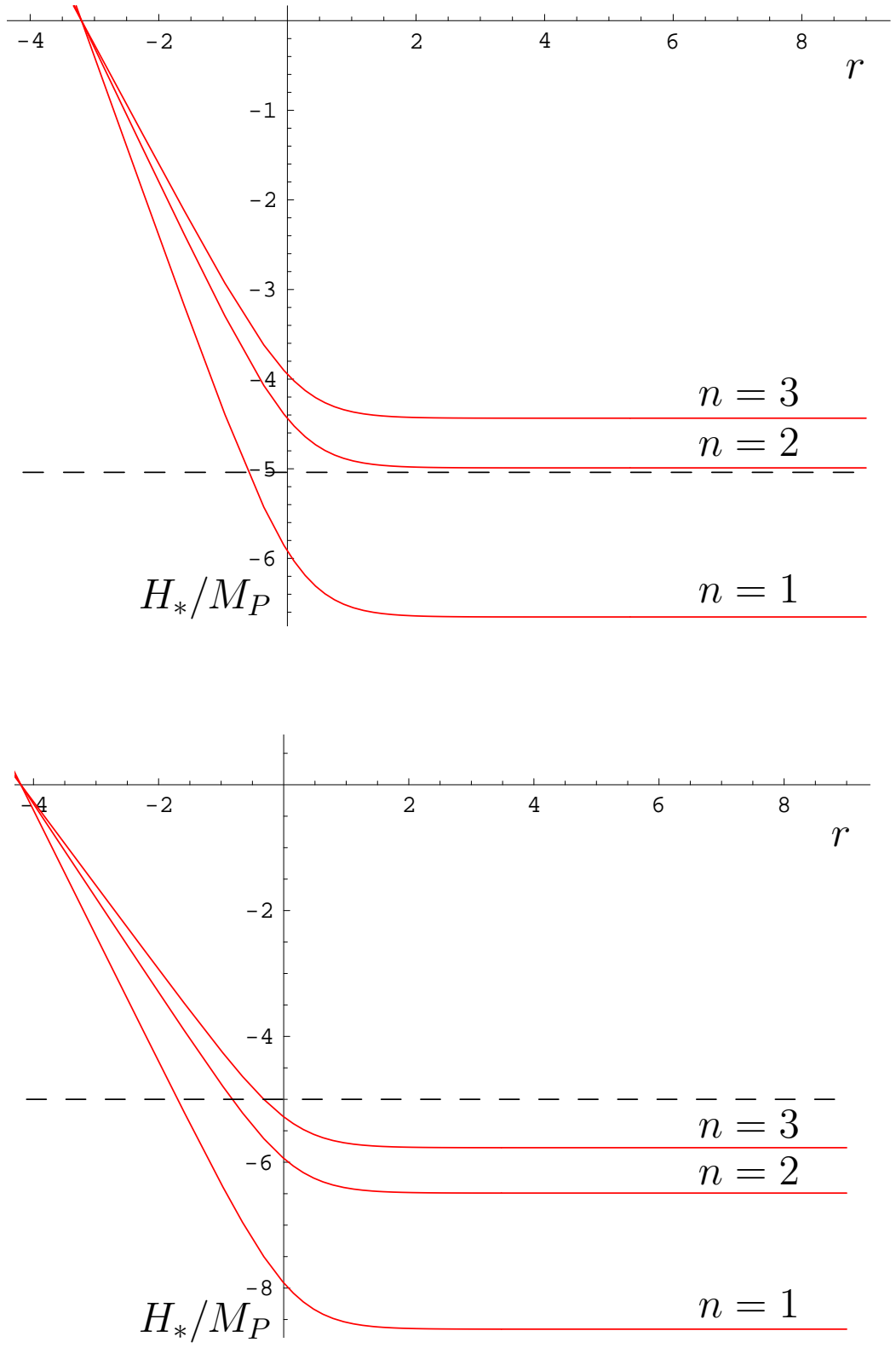

Figure 1: $\log -\log$ plots of $H_{*} / M_{P}$ with respect to $r$ for all cases: $n=1,2,3$. The dashed line corresponds to the bound: $H_{*} / M_{P}<10^{-5}$. The upper graph corresponds to $\theta_{0} \sim 1$, whereas the lower graph corresponds to $\theta_{0} \sim 0.1$. We see that, in the former case, only the case of $n=1$ manages to avoid the bound. On the other hand, in the latter case, all three possibilities are, in principle, allowed. The curves meet when $H_{*} \sim M_{P}$ as expected. 


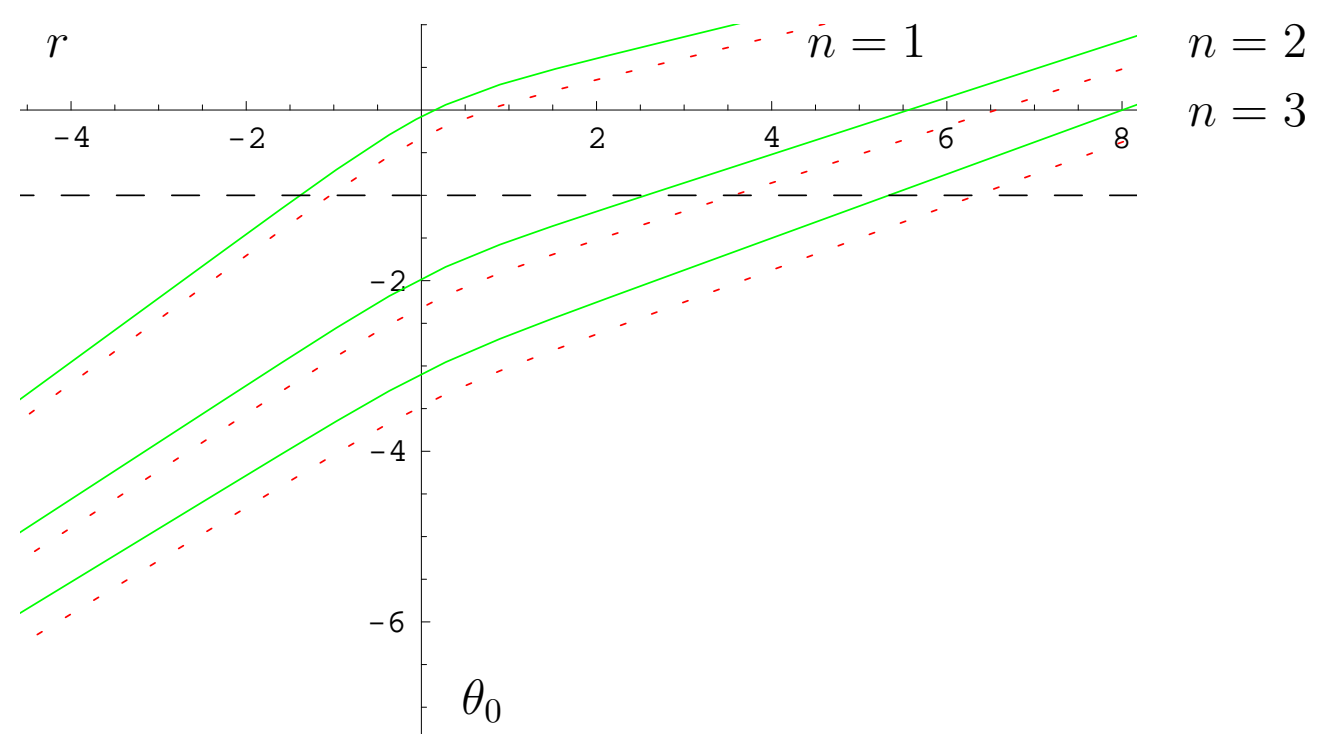

Figure 2: $\log -\log$ plot of $\theta_{0}$ with respect to $r$ for all cases $n=1,2,3$. The solid lines correspond to $H_{\bar{m}} \sim 1 \mathrm{GeV}$, whereas the dotted lines correspond to $H_{\bar{m}} \sim m_{3 / 2}$. The horizontal dashed line corresponds to $\theta_{0} \sim 0.1$. We see that, for $H_{\bar{m}}>1 \mathrm{GeV}$, the value of $r$ is slightly larger, for a given $\theta_{0}$ (it can increase up to an order of magnitude at most). Obviously, $\theta_{0}$ is bounded from above as $\log \theta_{0}<\log \pi \approx 0.5$. 


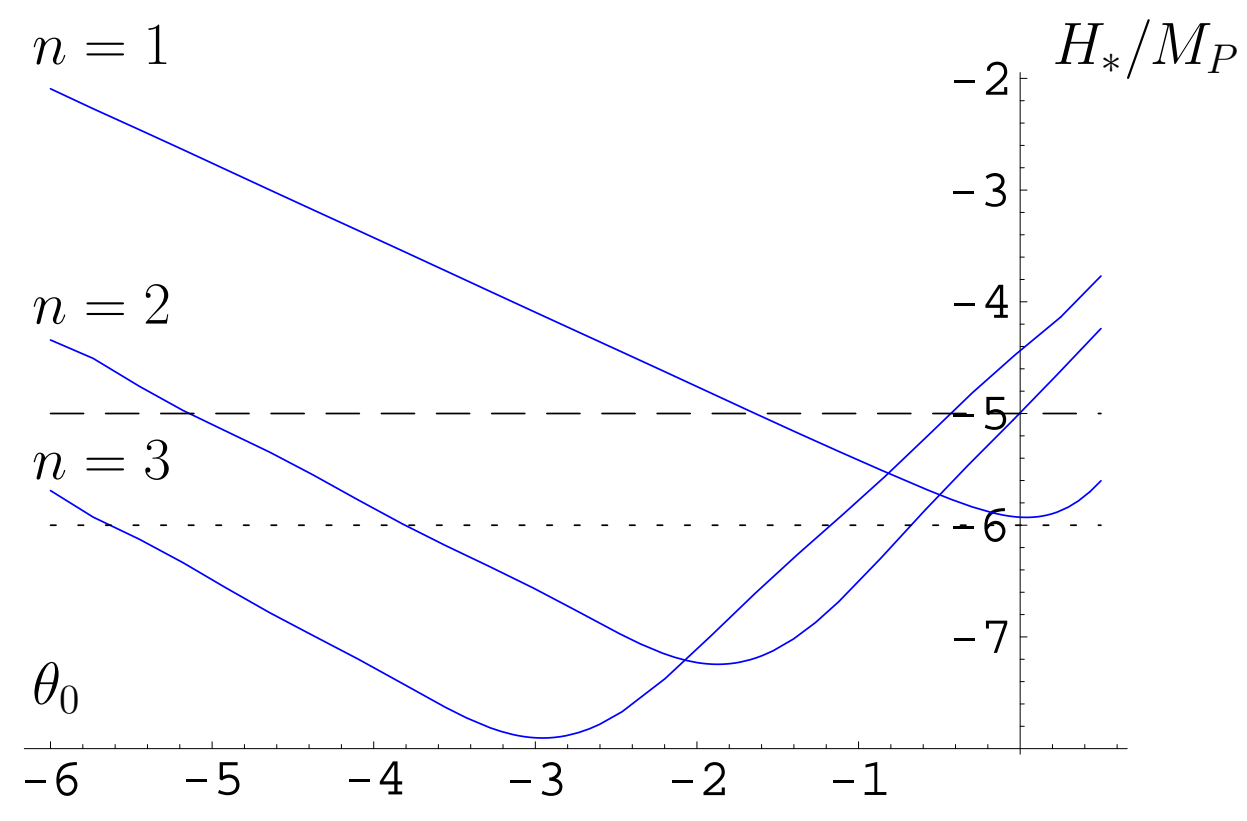

Figure 3: Log-log plots of $H_{*} / M_{P}$ with respect to $\theta_{0}$ for all cases: $n=1,2,3$ with $H_{\bar{m}} \sim 1 \mathrm{GeV}$. The dashed line corresponds to the bound: $H_{*} / M_{P}<10^{-5}$ (which ensures the compatibility with the observations), while the dotted line corresponds to the bound: $H_{*} / M_{P}<10^{-6}$ (which ensures that the inflaton does not contribute significantly to the curvature perturbations). We see that, when $\theta_{0} \sim 1$, only the case of $n=1$ is possible with $H_{*} / M_{P} \gtrsim 10^{-6}$, which means that the inflaton generated curvature perturbations are not negligible. For the curvaton to be the sole source of the curvature perturbations one has to limit oneself to the $n=2,3$ cases, where $\theta_{0} \ll 1$. 